\title{
Validated analytical RP-HPLC method for quantitation of wedelolactone from Eclipta alba and marketed Ayurvedic formulations
}

\author{
Sunita Shailajan ${ }^{1}$, Sasikumar Menon ${ }^{2}$, Dipti Singh ${ }^{1}$, Gauri Swar ${ }^{1}$ \\ 'Herbal Research Lab, Ramnarain Ruia College, Matunga (East), Mumbai-400019, INDIA. \\ Institute for Advanced Training and Research in Interdisciplinary Science, Plot No. 194, Scheme No. 6, Road No. 15, Sion Koliwada, Sion (East), Mumbai-400 022 , INDIA.
}

\begin{abstract}
Introduction: Eclipta alba Linn. (Asteraceae) is an important ingredient of several Ayurvedic formulations. The monograph on different parts of plant like flowers, leaves, roots are listed in Ayurvedic pharmacopeia of India. The plant is reported to be effective for broad range maladies like inflammation, reproductive problems of females etc. It is also used as a hepatoprotectant, analgesic, antibacterial and antidiabetic agent. Wedelolactone is used as a bioactive marker to establish the quality of the crude drug and its formulations. In the present study, wedelolactone-based standardization of Eclipta alba and its quantitation from marketed herbal and Ayurvedic formulations has been documented using RP-HPLC. Methods: In the current work, an isocratic method has been developed and validated to quantitate wedelolactone from whole plant of Eclipta alba. This method is validated as per $\mathrm{ICH}$ guidelines and is used to quantitate the content of wedelolactone in polyherbal formulations like Liv52, Geriforte, Mahabhringaraj oil etc. Results: The LOD is found to be $0.5 \mu \mathrm{g} / \mathrm{mL}$ and the $L O Q$ is $1 \mu \mathrm{g} / \mathrm{mL}$.
\end{abstract}

The linearity range of response is from $5 \mu \mathrm{g} / \mathrm{mL}$ to $100 \mu \mathrm{g} / \mathrm{mL}$. Conclusion: The validated method is found to be simple, sensitive, accurate, rugged and reproducible. This developed method can be recommended for quality assurance and marker-based standardization of polyherbal formulations containing whole plant of Eclipta alba.

Key words: Eclipta alba, Formulations, RP-HPLC, Validation, Whole plant, Wedelolactone.

Correspondence:

Dr. Sunita Shailajan, Associate Professor (Botany), Ramnarain Ruia College, Matunga (East), Mumbai-400 019, INDIA.

Tel no: 022 24154390, Fax. 02224142480

Email:-sunitashailajan@gmail.com

DOI: $10.5530 / p j .2016 .2 .6$

\section{INTRODUCTION}

Ayurveda 'the science of life' is a traditional system of medicine in India that dates back to ancient times. Ayurvedic preparations have been used to prevent diseases in both humans and animals for centuries in the Indian subcontinent; most of the formulations prepared by particular processing methods are compound preparations consisting of several herbs and are available in different forms such as powder, decoction, fresh juices, tablets, oils, ghee, ashavas and arishtas. Eclipta alba is one such herb used in various traditional and herbal formulations for various disorders.

Eclipta alba (L.) Hassk. Syn. E. erecta L. "Bhringaraja" is a small, branched annual herb with white flower heads. The plant grows abundantly as weed in waste places, marshy lands, hedges, roadsides and is widely distributed throughout India..$^{1-3}$ This herb is of ancient medicinal repute. In Ayurvedic system of medicine, it has been used for antimycotoxic, analgesic, antibacterial, antihepatotoxic, antihaemorrhagic, antihyperglycemic, antioxidant, immunomodulatory properties and is considered as a good rejuvenator too. ${ }^{4}$ The pharmacological activity of E. alba is related to several classes of secondary metabolites like coumestans, polypeptides, poly acetylenes, thiophene derivatives, steroids, triterpenes, wedelolactone, demethylwedelolactone, ursolic acid, $\beta$-sitosterol, stigmasterol, luteolin, stearic acid, oleanolic acid from whole plant of E. alba. ${ }^{5}$ Wedelolactone, a coumestan, (Figure 1), is the bioactive phytoconstituent from aerial parts of E. alba, which exhibits various biological activities such as hepatoprotective, antiplasmodial, sedative, anxiolytic, nootropic and anti-stress. ${ }^{6-8}$

E. alba is also an active ingredient of many herbal formulation prescribed for liver ailments and shows effect on liver cell generation. ${ }^{9}$ The expanded use of herbal medicines worldwide has led to concerns relating to its safety, quality and effectiveness. The quality control of herbal crude drugs and their formulations are of paramount importance in justifying their acceptability in modern system of medicine. One of the major problems faced by user industry is non-availability of rigid quality control profiles and evaluation parameters for herbal formulations.

Literature survey reveals that various chromatographic methods are available for quantitative estimation of wedelolactone. However, there are few HPTLC and HPLC methods available for analysis of wedelolactone from traditional formulations of varied matrices having short run length. ${ }^{10-11}$ Taking this objective in consideration, an attempt has been made to develop a simple, precise, rapid and effective HPLC method for the estimation of wedelolactone from whole plant of $E$. alba, their morphological parts, traditional and herbal formulation of varied matrices. Also, various extraction techniques have been tried and optimized to check for the presence of wedelolactone from these samples.

\section{MATERIALS AND METHODS}

\section{Plant Materials}

The flowering whole plant of E. alba was collected from Mumbai, Maharashtra and was authenticated by Agharkar Research Institute, Pune (Auth 1135/165) and a voucher specimen was deposited for further reference. The plant samples were also collected from different geographical regions of India like Thane, Alibaug, Mahabaleshwar, Vadodara, Kerala in order to study the impact of regional variation on the marker content. Samples were shade dried for 3 days, then dried at $37 \pm 2^{\circ} \mathrm{C}$ for 2 days, powdered, sieved through BSS sieve (85-mesh) and stored in air-tight containers.

\section{Reference standards and reagents}

Wedelolactone (Purity $>95 \%$ ) was purchased from Natural Remedies Pvt Ltd, Bangalore. HPLC grade solvents were procured from Merck Special- 


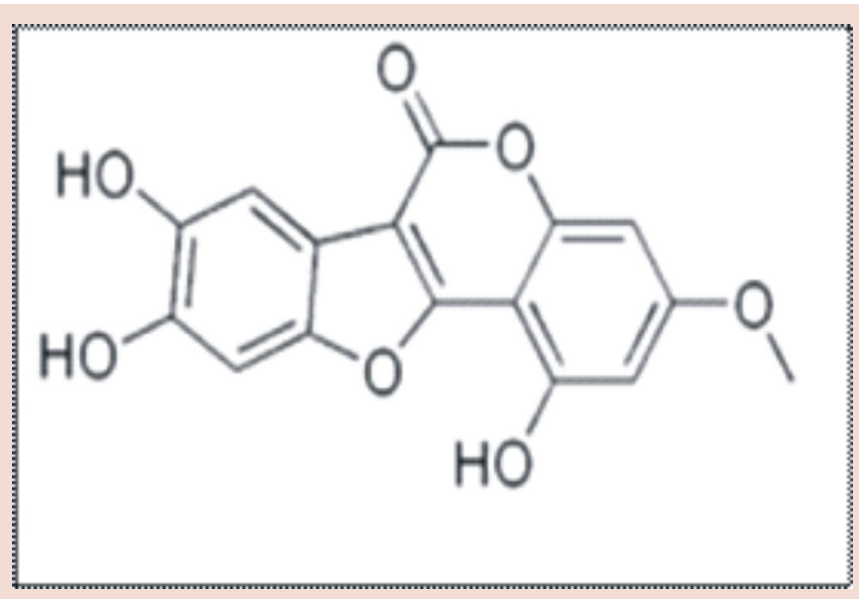

Figure 1: Structure of Wedelolactone

ties Pvt. Ltd., Mumbai, India. All other chemicals used were of Analytical grade.

\section{Marketed formulations}

Various traditional Ayurvedic and herbal marketed formulations of varied matrices containing E. alba as one of the ingredient like Mahavatvidhwansan Ras (Baidyanath, Batch No. 130223), Keshkuntal (Vyas Pharmaceuticals, Batch No. 00109), Bhrungraj Ghana (Chaitanya Pharmaceuticals Pvt Ltd, Batch No. CP1403), Marcal (SEAMCO, Batch No. 13-5), Liv 52 (The Himalaya Drug Company, Batch No. 30049011), Purim (The Himalaya Drug Company, Batch No. 37301735B), Mahabhringraj oil (Ramkrishna Vidyut Ayurvedic Pharmacy, Batch No. 2219), Geriforte syrup (The Himalaya Drug Company, Batch No. 25300457) and Pilex ointment (The Himalaya Drug Company, Batch No. 20400415) were purchased from local market (Mumbai) and was used for the quantification of wedelolactone.

\section{Preparation of standard solution}

Standard stocksolutions $(1000 \mu \mathrm{g} / \mathrm{mL})$ of wedelolactone was prepared in methanol. Working standard solutions of wedelolactone was prepared and diluted with methanol to obtain appropriate concentrations for establishment of calibration curves $5-100 \mu \mathrm{g} / \mathrm{mL}$. Three quality control samples (LQC-low, MQC-mid, HQC-high) of wedelolactone $(7,23,80 \mu \mathrm{g} / \mathrm{mL})$ were prepared for method validation studies and stored at $4^{\circ} \mathrm{C}$.

\section{Optimized Extraction Conditions}

\section{Extraction condition for different samples of $E$. alba}

The powder of E. alba (whole plant) collected from Sion ( $0.5 \mathrm{~g}$ each), Mahabaleshwar $(0.5 \mathrm{~g})$, Varanasi $(0.2 \mathrm{~g})$ and the samples collected from other regions ( $0.1 \mathrm{~g}$ each), different morphological parts such as root $(0.5 \mathrm{~g}$ each), stem $(0.5 \mathrm{~g}$ each $)$, leaves $(0.1 \mathrm{~g}$ each $)$ and flowers $(0.1 \mathrm{~g}$ each) collected from Mahabaleshwar were extracted in methanol $(10 \mathrm{~mL})$ into separate stoppered test tubes. The mixtures were vortexed for 1 minute, kept standing overnight, sonicated for $5 \mathrm{~min}$ and filtered through Whatman filter paper no.1 followed by filtration through millipore filter $(0.45 \mu \mathrm{m})$ and $20 \mu \mathrm{L}$ of the sample was used for further HPLC analysis.

\section{Extraction conditions for marketed formulations}

Polyherbal formulations of varied matrices like tablets, oil, syrup, ointment were analyzed for the presence of wedelolactone from them. Tablets like Mahavatvidhwansan Ras (MR), Keshkuntal (K), Bhrungraj Ghana (BG), Marcal (M), Liv 52 (L), Purim (PU), syrup (Geriforte - GF), oil based formulation (Mahabhringaraj oil-MT), ointment (Pilex-PI) were extracted using different extraction techniques like overnight extraction, shaker, reflux. The technique which was most suitable for the extraction of phytochemical marker from the sample was selected.

Tablets: The tablets were crushed using mortar and pestle after removing the outer coat. Powder of Keshkuntal (4g), Mahavatvidhwansan Ras (2g) and $(0.5 \mathrm{~g})$ for other tablets were extracted in methanol $(10 \mathrm{~mL})$ into stoppered test tube.

Oil based formulation: Mahabhringaraj oil $(2 \mathrm{~mL})$ was extracted in methanol $(3 \mathrm{~mL})(\mathrm{v} / \mathrm{v})$ into stoppered conical flask. The mixture was vortexed for $1 \mathrm{~min}$ and kept on the shaker for $6 \mathrm{~h}$ at $65 \mathrm{rpm}$ followed by overnight refrigeration at $4^{\circ} \mathrm{C}$. Next day, the immiscible organic layer was separated, filtered through whatman filter paper no.1 followed by filtration through millipore filter $(0.45 \mu \mathrm{m})$ and used for further HPLC analysis.

Liquid formulation: Geriforte $(2 \mathrm{~mL})$ was extracted in chloroform $(3 \mathrm{~mL})$. The mixture was vortexed for 1 minute and refluxed for $6 \mathrm{~h}$ at $30 \%$ heat. The immiscible organic layer was separated and evaporated to dryness at $45^{\circ} \mathrm{C}$ and reconstituted with $200 \mu \mathrm{L}$ of methanol. The sample was filtered through millipore filter $(0.45 \mu \mathrm{m})$ and used for further HPLC analysis.

\section{Ointment based formulation}

Extraction procedure 1: Pilex Ointment (2 g) was extracted in methanol $(5 \mathrm{~mL})$. The mixture was vortexed for 1 minute and kept for overnight extraction at room temperature for $15 \mathrm{~h}$. On next day, the mixture was filtered through Whatman filter paper no. 1 followed by filtration through millipore filter $(0.45 \mu \mathrm{m})$ and used for further HPLC analysis.

Extraction procedure 2: Ointment $(2 \mathrm{~g})$ was extracted in methanol $(5 \mathrm{~mL})$ in stoppered conical flask. The mixture was vortexed for 1 minute and kept on the shaker for $6 \mathrm{~h}$ at $65 \mathrm{rpm}$ and then sonicated for $10 \mathrm{mins}$ in sonicator. The mixture was then filtered through Whatman filter paper no. 1 followed by filtration through millipore filter $(0.45 \mu \mathrm{m})$ and used for further HPLC analysis.

Extraction procedure 3: Ointment $(2 \mathrm{~g})$ was extracted in methanol $(5 \mathrm{~mL})$. The mixture was vortexed for $1 \mathrm{~min}$ and refluxed for $6 \mathrm{~h}$ at $30 \%$ heat. The mixture was filtered through Whatman filter paper no. 1 followed by filtration through millipore filter $(0.45 \mu \mathrm{m})$ and used for further HPLC analysis.

\section{Instrumentation and Optimized Chromatographic Conditions}

\section{High Performance Liquid Chromatography (HPLC)}

Chromatographic analysis was performed at room temperature using Jasco's HPLC system comprising of two PU-1580 pumps (HG-1580-31), rheodyne injector (20 $\mu \mathrm{L}$ loop) and Photo Diode Array (PDA) detector (MD-1510). Chromatograms were recorded by means of Jasco-Borwin chromatography software version 1.50. Separation was achieved on Cosmosil $\mathrm{C}_{18}$-column $(150 \times 4.6 \mathrm{~mm}, 5 \mu \mathrm{m}$ particle size $)$ using methanol: $0.2 \%$ formic acid in water $(57: 43 \mathrm{v} / \mathrm{v})$, delivered at a flow rate of $1 \mathrm{~mL} /$ min. After, $10 \mathrm{~min}$ of equilibration period, the samples were injected into HPLC system. Injection volume was $20 \mu \mathrm{L}$ and the elute was analyzed at a wavelength of $351 \mathrm{~nm}$.

\section{Validation of the method}

The analytical method was validated for linearity, accuracy, precision, limit of detection (LOD), limit of quantification (LOQ), specificity, robustness and ruggedness, in accordance with ICH guidelines. Further calculations were performed. 


\section{Selectivity and specificity}

During the experiment, UV scan ranging from $200-400 \mathrm{~nm}$ in the time window of the analytes using PDA detector was performed with the aim of revealing eventual interfering compounds and evaluating the selectivity of the method. Specificity of the intended method was established by comparing the HPLC retention time and absorption spectra of target peaks from the analyzed samples with that of the reference standard.

\section{System suitability}

System suitability experiment was performed by injecting five consecutive injections of the marker wedelolactone $(5 \mu \mathrm{g} / \mathrm{mL})$. Values with $\% \mathrm{CV}$ of $\leq 2 \%$ were accepted.

\section{Calibration curves}

Linearity of wedelolactone was determined at seven different concentrations $(5-100 \mu \mathrm{g} / \mathrm{mL})$ in triplicate and plotted using linear regression of the mean peak area versus concentration. The linear regression equation was obtained to estimate the content of wedelolactone from the analyzed samples.

\section{Limits of detection (LOD) and quantification (LOQ)}

Sensitivity of the method was evaluated by determining the values of LOD and LOQ. Stock solution of each standard was serially diluted with methanol to prepare the series of samples with least concentration and injected into the HPLC system. The limit of detection (LOD) and quantification (LOQ) for wedelolactone were determined by measuring the signal to noise ratio $(\mathrm{S} / \mathrm{N})$. LOD and LOQ was considered at $\mathrm{S} / \mathrm{N}$ of $3: 1$ and 10:1 respectively.

\section{Precision}

Precision was studied by measuring intra-day (repeatability) and inter-day (by injection of samples over three consecutive days). Intra-day precision was evaluated from replicate analysis $(n=5)$ of quality control samples on the same day while inter-day precision was assessed by analyzing them on three consecutive days $(n=5)$. The $\% \mathrm{CV}$ was taken as a measure of precision. Accuracy values within the range of $85-115 \%$ and $\% \mathrm{CV}$ of $\leq$ $2 \%$ were accepted.

\section{Stability}

Stock solution stability of wedelolactone was tested at room temperature and samples were analyzed in triplicate after 15 days. Same samples in triplicate were also subjected to bench top stability testing at $27 \pm 2^{\circ} \mathrm{C}$ for $0 \mathrm{~h}$ and $6 \mathrm{~h}$ respectively. Values within a difference range of $\pm 5 \%$ were accepted.

\section{Robustness and Ruggedness}

The robustness of the method was investigated by making small deliberate changes in the chromatographic conditions at three different levels. In brief, four factors which could influence the result were chosen. The factors were HPLC column [columns of two different lot/batch from same manufacturer (Cosmosil, K64003 and K65110)], analyst (analyst 1 and 2$)$, flow rate $(1 \pm 0.1 \mathrm{~mL} / \mathrm{min})$ and mobile phase composition [methanol $(57 \pm 1): 0.2 \%$ formic acid in water $(43 \pm 1)$, (v/v)]. Effect of these deliberate changes on the response (area) and retention time of QC samples of wedelolactone was observed during the analysis. The results were expressed in terms of $\%$ mean difference. Values within a difference range of $\pm 5 \%$ were accepted.

\section{Recovery}

Recovery of the method was evaluated using standard addition method. It was evaluated in triplicate, by adding a known amount of wedelolactone standard (LQC, MQC, HQC) to a sample of known concentration and calculating the recovery of wedelolactone for each concentration.
The mean percent recovery was calculated using the formula: Recovery $(\%)=[($ amount found - original amount $) /$ amount added $] \times 100$. Values within the range of $95-105 \%$ were accepted.

\section{Estimation of wedelolactone from the different samples of E. alba and formulations}

Relative retention time and relative peak area of each characteristic peak from the samples of E. alba related to the peak from wedelolactone were calculated for the estimation of wedelolactone content from the samples of E. alba collected from different geographical regions, their morphological parts, traditional and herbal formulations of varied matrices using the regression equation.

\section{Statistical evaluation}

Microsoft Excel 2007 was used to determine mean, standard deviation, relative standard deviation and mean difference during the analysis.

\section{RESULTS}

\section{Mobile phase development}

The primary aim of the present study was to separate wedelolactone from various compounds in E. alba extracts and herbal formulations. This included mobile phase selection, flow rate and column type. In this experiment, mixtures of several mobile phases were tried to separate peak of wedelolactone from other peaks and get a stable peak. The mobile phase, methanol: $0.2 \%$ formic acid in water in isocratic elution with a flow rate of $1 \mathrm{~mL} / \mathrm{min}$ resulted in good resolution, symmetrical peak shape of wedelolactone and short analysis time per run. All the samples exhibited the UV maximum absorption at $351 \mathrm{~nm}$ corresponding to the standard wedelolactone. All the reference compounds were easily resolved in the optimized HPLC condition and were eluted within $10 \mathrm{~min}$. The retention time was $5.9 \mathrm{~min}$ for standard as well as the samples. The suitability of the solvent system was decided by cost, sensitivity of the assay and time required for the analysis. A chromatogram of standard wedelolactone has been shown in Figure 2. Hence, the developed method was found to be time effective when compared with the other published report.

\section{Method validation}

\section{Selectivity and specificity}

During the UV scan, no appreciable difference was found in the spectra of reference standards and the analyzed samples. Hence the method demonstrated a high degree of selectivity.

\section{System suitability}

System suitability tests are an integral part of liquid chromatographic method. They are used to verify whether the resolution and reproducibility of the chromatographic system are adequate for the analysis. For wedelolactone, the $\% \mathrm{CV}$ for retention time was $1.71 \%$ and $1.34 \%$ for response respectively.

\section{Linearity}

Seven point standard curves were obtained by plotting the peak area versus concentration. Calibration curves were found to be linear over the concentration range studied. Mean correlation coefficient (coefficient of determination, $\mathrm{r}^{2}$ ) was found to be 0.999 , during the course of validation indicating positive linear relationship between peak area and concentration. Results of regression analysis are summarized in Table 1.

\section{Sensitivity}

Sensitivity of the method was affirmed in terms of limit of detection (LOD) and limit of quantification (LOQ) for wedelolactone and the 
Table 1: Method validation data for determination of wedelolactone

\begin{tabular}{cc|}
\hline Parameters & Value \\
\hline Mean retention time & $5.9 \mathrm{~min}$ \\
Linear range & $5-100 \mu \mathrm{g} / \mathrm{mL}$ \\
Equation & $\mathrm{y}=31.705 \mathrm{x}-32261$ \\
Regression coefficient & 0.999 \\
Limit of Detection (LOD) & $0.5 \mu \mathrm{g} / \mathrm{mL}$ \\
Limit of Quantitation (LOQ) & $1.0 \mu \mathrm{g} / \mathrm{mL}$ \\
Validated LQC & $7.0 \mu \mathrm{g} / \mathrm{mL}$ \\
Validated MQC & $23.0 \mu \mathrm{g} / \mathrm{mL}$ \\
Validated HQC & $80.0 \mu \mathrm{g} / \mathrm{mL}$ \\
Recovery & $98.8 \%$ \\
\hline
\end{tabular}

Table 2: Precision of the proposed method

\begin{tabular}{ccccc} 
Parameters & $\begin{array}{r}\text { Repeatability (Intraday } \\
\text { precision)* }\end{array}$ & $\begin{array}{c}\text { Intermediate precision } \\
\text { (Inter day precision)* }\end{array}$ \\
\hline Concentration & $\% \mathrm{CV}$ & $\%$ Nominal & $\% \mathrm{CV}$ & $\%$ Nominal \\
LQC & 1.11 & 100.25 & 1.61 & 101.46 \\
MQC & 1.59 & 92.39 & 1.76 & 94.11 \\
HQC & 0.26 & 90.25 & 1.84 & 89.44 \\
\hline * & & & &
\end{tabular}

${ }^{\star}$ Mean $\pm \mathrm{SD}, \mathrm{n}=5$

Table 3: Stability of the solutions at various parameters

\begin{tabular}{|c|c|c|c|c|c|c|c|}
\hline \multirow[t]{2}{*}{ Parameters } & \multicolumn{2}{|c|}{$\begin{array}{l}\text { Bench top stability } \\
\left.\text { (at } 25 \pm 20^{\circ} \mathrm{C} \text { for } 2 \mathrm{~h}\right)^{*}\end{array}$} & \multicolumn{2}{|c|}{$\begin{array}{l}\text { Short term stability } \\
\left.\text { (at } 4 \pm 10^{\circ} \mathrm{C} \text { for } 6 \mathrm{~h}\right)^{*}\end{array}$} & \multicolumn{3}{|c|}{$\begin{array}{l}\text { Long term stability } \\
\text { (at }<8^{\circ} \mathrm{C} \text { for } 7 \text { and } 14 \text { days)* }\end{array}$} \\
\hline & BTS 01 & BTS 02 & STS 01 & STS 02 & LTS 01 & LTS 02 & LTS 03 \\
\hline$R_{t}(\% C V)$ & 2.00 & 0.81 & 1.90 & 1.85 & 0.96 & 0.84 & 0.53 \\
\hline Area (\% CV) & 1.82 & 0.82 & 0.60 & 1.07 & 1.15 & 1.21 & 1.78 \\
\hline
\end{tabular}

${ }^{*}$ Mean $\pm S D, n=5$.

Table 4: Ruggedness of the method

\begin{tabular}{|c|c|c|c|c|c|c|c|c|c|c|c|}
\hline \multirow{2}{*}{\multicolumn{2}{|c|}{ Parameters }} & \multicolumn{3}{|c|}{ Change in mobile phase $( \pm 1 \%)^{*}$} & \multicolumn{3}{|c|}{ Change in flow rate $( \pm 0.1 \mathrm{~mL})^{*}$} & \multicolumn{2}{|c|}{ Change in Analyst* } & \multicolumn{2}{|c|}{$\begin{array}{l}\text { Change in batch of } \\
\text { Column)* }\end{array}$} \\
\hline & & MP1 & MP 2 & MP 3 & FR 1 & FR 2 & FR 3 & ANA 1 & ANA 2 & COL 1 & COL 2 \\
\hline \multirow{2}{*}{ LQC } & $\mathrm{R}_{\mathrm{t}}(\% \mathrm{CV})$ & 0.72 & 1.20 & 1.06 & 1.23 & 1.53 & 1.83 & 1.34 & 0.27 & 1.45 & 0.37 \\
\hline & Area $(\% \mathrm{CV})$ & 1.38 & 1.55 & 1.17 & 0.71 & 0.31 & 0.40 & 1.43 & 1.63 & 1.83 & 1.48 \\
\hline \multirow{2}{*}{ MQC } & $\mathrm{R}_{\mathrm{t}}(\% \mathrm{CV})$ & 1.70 & 0.38 & 1.94 & 0.80 & 1.61 & 1.06 & 1.67 & 1.72 & 0.54 & 1.06 \\
\hline & Area $(\% \mathrm{CV})$ & 1.80 & 1.51 & 0.91 & 1.69 & 1.98 & 1.29 & 0.54 & 1.30 & 0.15 & 0.73 \\
\hline \multirow{2}{*}{ HQC } & $\mathrm{R}_{\mathrm{t}}(\% \mathrm{CV})$ & 0.99 & 1.54 & 0.44 & 0.62 & 1.48 & 1.19 & 1.01 & 0.35 & 0.82 & 0.70 \\
\hline & Area (\% CV) & 0.74 & 1.17 & 0.65 & 1.51 & 2.00 & 1.08 & 0.42 & 0.09 & 1.74 & 1.82 \\
\hline
\end{tabular}

${ }^{*}$ Mean $\pm \mathrm{SD}, \mathrm{n}=5$

results are represented in Table 1 . The values for both $\mathrm{LOD}(0.5 \mu \mathrm{g} / \mathrm{mL})$ and LOQ $(1 \mu \mathrm{g} / \mathrm{mL})$ were low when compared to other published work ${ }^{12}$ which indicated that the method can be effectively used in a wide range for detection and quantification of wedelolactone from plant samples. The low values for LOD and LOQ indicate the sensitivity of the proposed method.

\section{Precision}

Precision was considered at two different levels (i.e. repeatability and intermediate precision). Intra-day variation was carried out to determine repeatability of sample application whereas intermediate precision was determined by carrying out inter-day variation using replicate injection $(\mathrm{n}=5)$ of quality control samples (LQC, MQC, HQC) of wedelolactone. The results of repeatability and intermediate precisionwere expressed in terms of $\% \mathrm{CV} \leq 2 \%$ and $\%$ accuracy of $89.44-101.46 \%$ and are shown in Table 2.

\section{Stability}

Stock solution stability study of wedelolactone samples stored for the period of 15 days at $4^{\circ} \mathrm{C}$ showed $\% \mathrm{CV}<2 \%$ with the $\%$ mean difference within $\pm 5 \%$. During bench top stability study similar results were obtained. Stability studies showed that quality control samples of wedelolactone were stable for at least $6 \mathrm{~h}$ at room temperature and 15 days at $4^{\circ} \mathrm{C}$ of storage condition. (Results are summarized in Table 3).
Recovery

Recovery experiment afforded the average recovery of 101.59, 97.32 and 97.53\% for LQC, MQC and HQC respectively, after spiking the additional standard (quality control samples) solution to the previously analyzed plant matrix following the extraction procedure.

\section{Ruggedness}

Proposed method was not influenced by the four factors considered in ruggedness study. Small but deliberate changes in the method parameters like change in column and analyst did not affect the retention time and response of wedelolactone samples since, $\% \mathrm{CV}(\leq 2 \%)$ and $\%$ mean difference $( \pm 5 \%)$ were within the acceptance criteria. Change in the flow rate and mobile phase composition affected the retention time of wedelolactone but the results were satisfactory since $\% \mathrm{CV}$ was $<2 \%$ with a \%mean difference of $\pm 5 \%$. Results are summarized in Table 4 .

\section{Assay and method application}

The developed method was used as an application to determine the presence of wedelolactone from samples of E. alba collected from various geographical regions and from their morphological plant parts and from the marketed herbal and Ayurvedic formulations of varied matrices. 


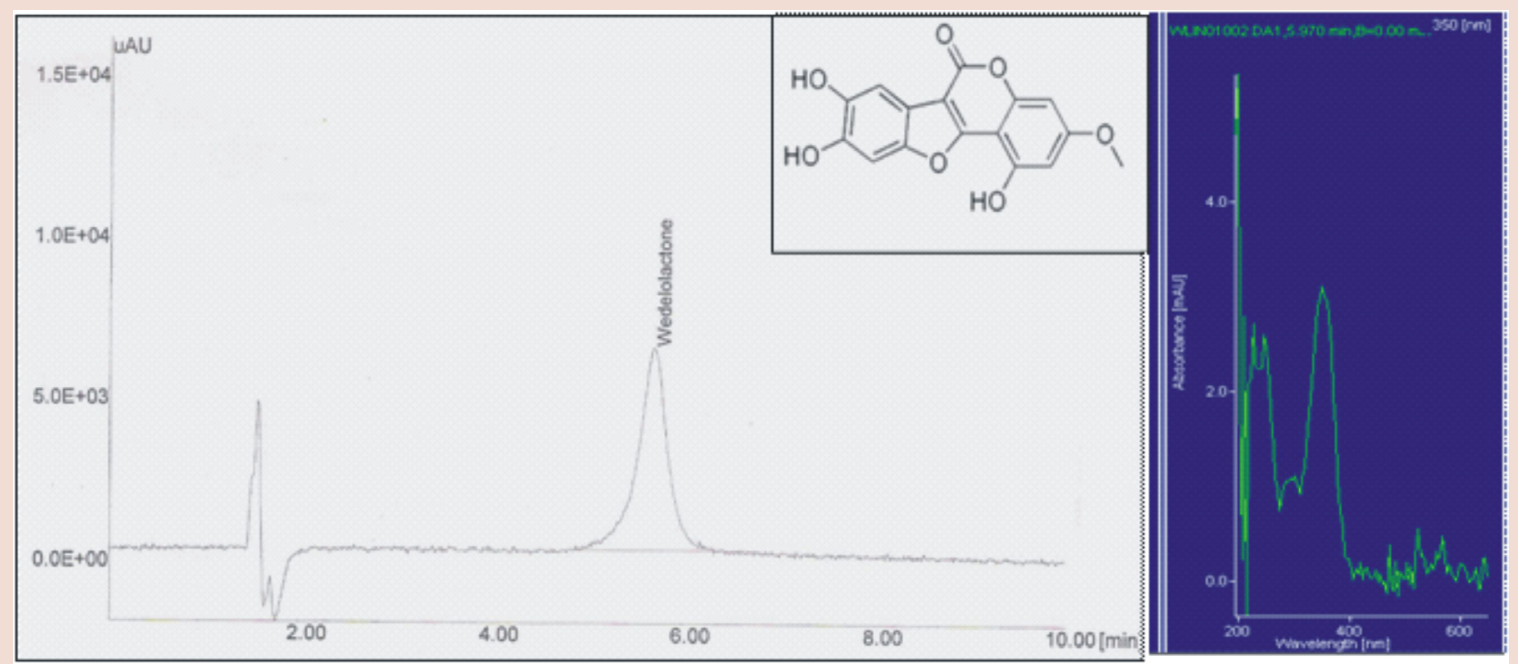

Figure 2: HPLC Chromatogram of Standard Wedelolactone $(5 \mu \mathrm{g} / \mathrm{mL})$
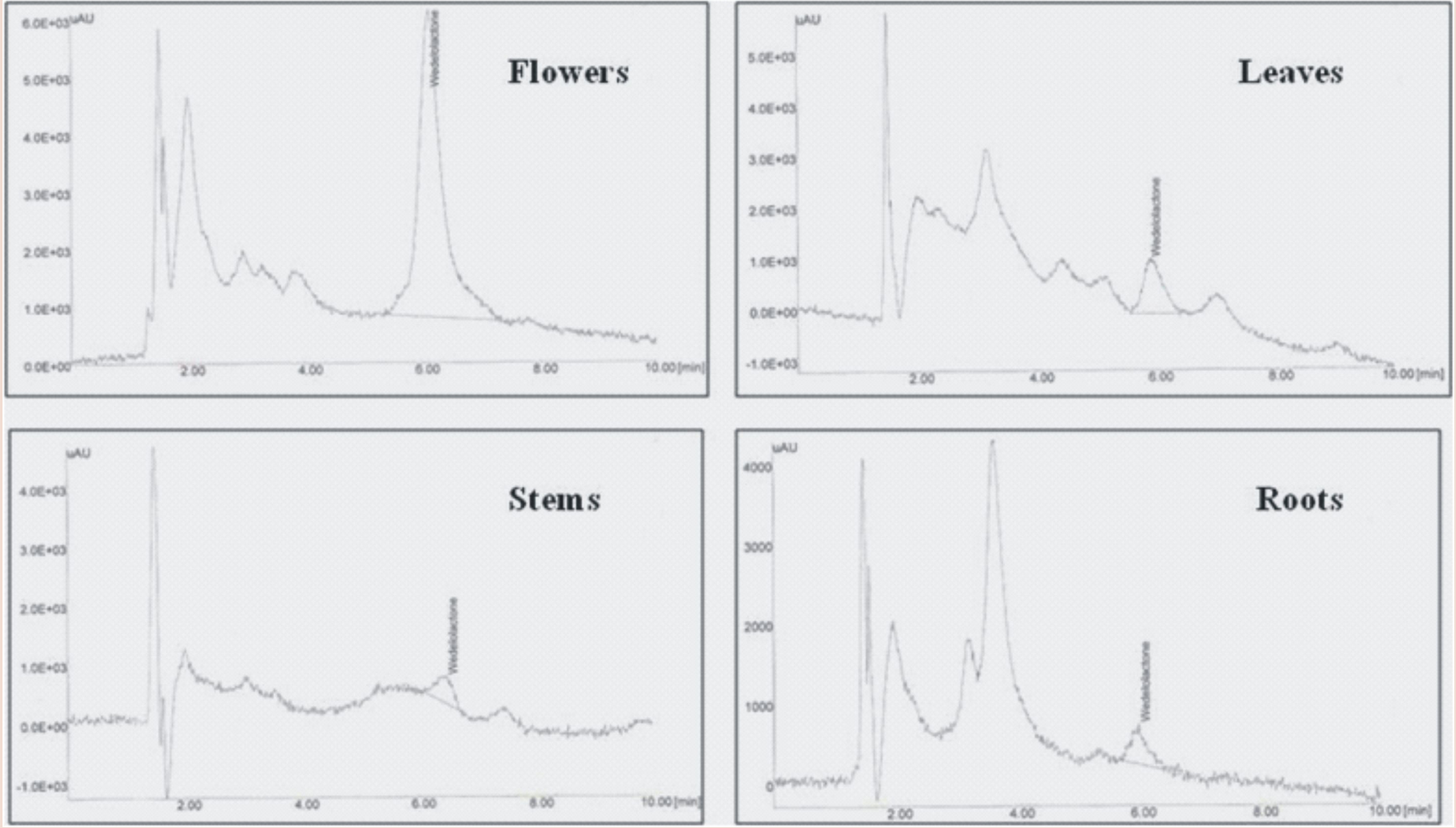

Figure 3: HPLC Chromatogram of different morphological parts of Eclipta alba

Estimation of wedelolactone from the whole plant extract of E. alba collected from different geographical regions of India and their morphological parts

The method was finally tested to evaluate the content of wedelolactone from whole plant of $E$. alba collected from various geographical regions of India and from different morphological parts of E. alba, using regression equation. The peak of wedelolactone from different samples were identified by comparing their retention times obtained from the peak of standard
(Figure 3). HPLC profile of methanolic extract of E. alba was developed through the same conditions as it was for the standard. It was observed that the sample collected from Alibaug showed maximum content of wedelolactone followed by samples collected from Kerala, Thane, Vadodara, Mahabaleshwar and Mumbai region. Results are summarized in Table 5. 

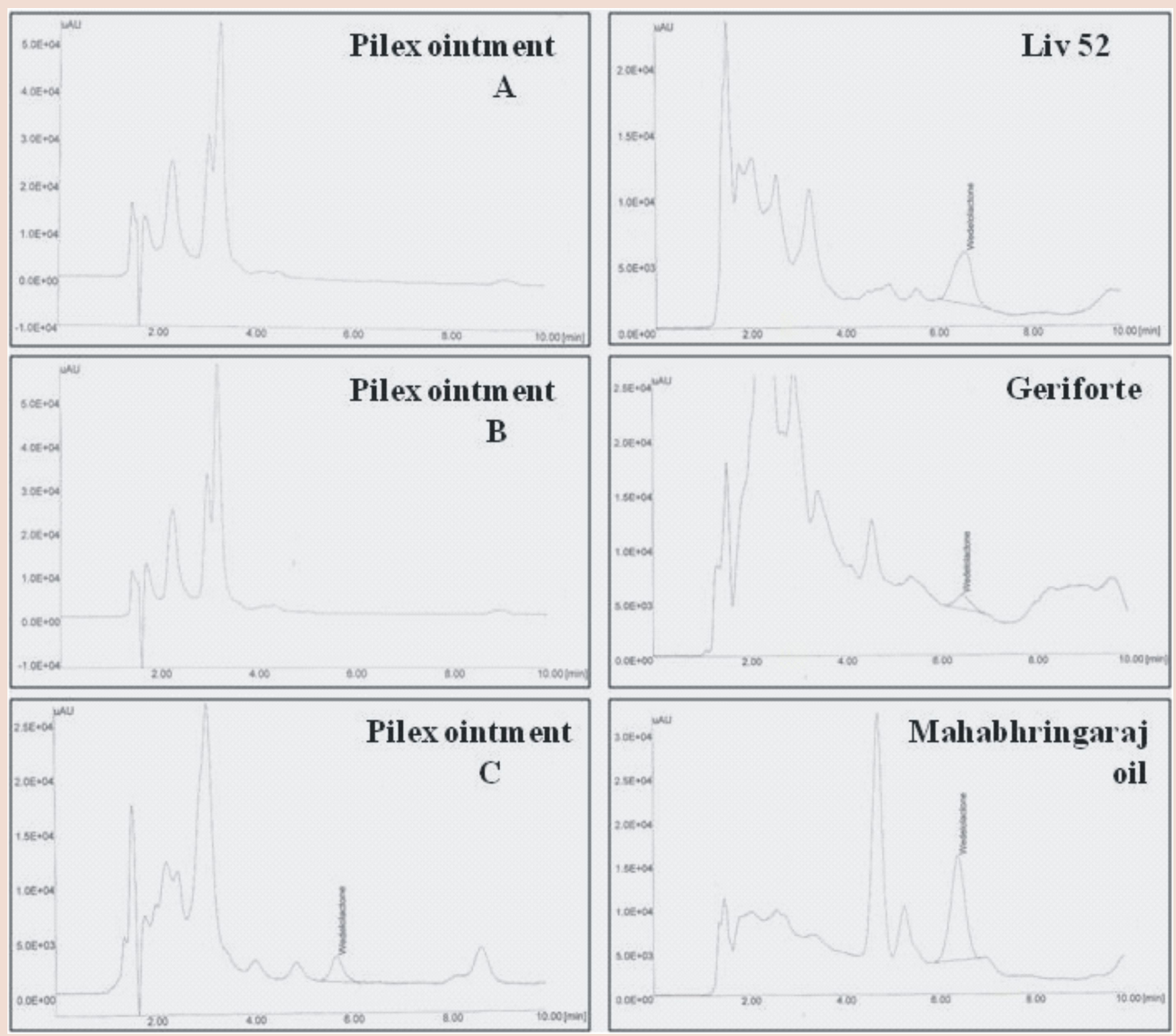

Figure 4: HPLC Chromatogram of various traditional and herbal formulations of Eclipta alba

Different morphological parts of E. alba collected from the same plant were also used for the determination and estimation of marker content. The same extraction technique was used to extract wedelolactone from different parts of E. alba. Wedelolactone was found to be present in all the four parts with highest content in the flowers followed by leaves, stem and roots which is evident from Table 5.

\section{Estimation of wedelolactone from different marketed Ayurvedic and herbal formulations containing E. alba}

Different Ayurvedic and herbal formulations containing E. alba as one of their ingredient were tried for the presence of Wedelolactone in it using HPLC. Different extraction techniques were tried for the formulations with varied matrices. A single peak was observed at the same retention time in the samples of formulations (Figure 4). There was no interaction between wedelolactone and other excipients present in the marketed Ayurvedic and herbal formulations. Formulations of varied matrices like tablets, oil, syrup and ointment were tried for the presence of wedelolac- tone. Among the various formulations, Liv 52, a polyherbal formulation in the form of tablet showed the maximum content of Wedelolactone. The content of marker was found to be least in Keshkuntal amongst all the solid formulations used. The content of the wedelolactone in the formulations was found to be less as compared with the expected content as per their label claim. This variation could be attributed to the amount of crude drug added or the quality of the material used by the industry.

The results obtained from Mahavatvidhwansan Ras are with par to the expected value justifying the label claim. The content of wedelolactone in Mahabhringaraj oil, Geriforte, Pilex was found to be $0.0027 \mathrm{mg} / \mathrm{mL}$, $0.0017 \mathrm{mg} / \mathrm{mL}$ and $0.0011 \mathrm{mg} / \mathrm{g}$ respectively. Between the two liquid preparations used, Mahabhringaraj oil which is an oil showed higher marker content as compared to the water based syrup Geriforte, which is summarized in Table 5. This might be due to the difference in the extraction techniques as well as extracting solvents used for both the formulation. Oil based formulation (Mahabhringaraj oil) was extracted directly in methanol whereas the liquid based formulation $(G)$ was fractionated 
with chloroform. The detection of higher marker content in the oil may be due to the better extraction efficiency of methanol for wedelolactone as compared to chloroform which can be attributed to the higher solubility of wedelolactone in methanol as compared to chloroform.

The effect of extraction was also seen in the ointment based formulation, Pilex. All the three samples used were same but extracted using different extraction techniques. It was observed that the first two techniques of extraction i.e overnight extraction and extraction using shaker (Figure $4 \mathrm{a}$ and $4 \mathrm{~b}$ ) failed to extract wedelolactone from the matrix, but the third technique of using reflux (Figure 4c) was able to extract the standard to a quantifiable range (Table 5).

\section{Table 5: Content of Eclipta alba in different samples}

\begin{tabular}{cc}
\hline Samples & Content $(\mathrm{mg} / \mathrm{g})^{*}$ \\
\hline Geographical variation & $0.0337 \pm 0.0020$ \\
Thane & $0.0078 \pm 0.0016$ \\
Mumbai & $0.0104 \pm 0.0039$ \\
Alibaug & $0.0974 \pm 0.0041$ \\
Vadodara & $0.0318 \pm 0.0034$ \\
Kerala & $0.0398 \pm 0.0008$ \\
Marketed crude sample No.1 (Mumbai) & $0.1522 \pm 0.0213$ \\
Marketed crude Sample No.2 (Varanasi) & $0.1106 \pm 0.0134$ \\
Morphological variation & \\
Roots & $0.0018 \pm 0.0009$ \\
Stems & $0.0026 \pm 0.0011$ \\
Flowers & $0.0587 \pm 0.0028$ \\
Leaves & $0.0130 \pm 0.0036$ \\
Herbal and Ayurvedic Formulations & \\
Mahavatvidhwansan Ras tablets & $0.0026 \pm 0.0001$ \\
Keshkuntal tablets & $0.0006 \pm 0.00001$ \\
Bhrungraj Ghana tablets & $0.0072 \pm 0.0018$ \\
Marcal tablets & $0.0069 \pm 0.0009$ \\
Liv 52 tablets & $0.0180 \pm 0.0009$ \\
Purim tablets & $0.0079 \pm 0.0007$ \\
Mahabhringaraj Oil & $0.0027 \pm 0.0002$ \\
Geriforte syrup & $0.0017 \pm 0.0011$ \\
Pilex ointment & $0.0011 \pm 0.0031$ \\
\hline Mabil &
\end{tabular}

${ }^{\star}$ Mean $\pm S D, n=3$.

\section{DISCUSSION}

Plant constituents vary considerably depending on several factors like temperature, light, drying, packing, storage etc. These variations impair not only the quality of phytotherapeutic agents but also their therapeutic value. The source and quality of raw materials play a pivotal role in ensuring the quality and stability of herbal preparations. Thus, standardization and quality control of raw material and the herbal preparations themselves need to be permanently carried out.

In the development of the HPLC method for the estimation and quantitation of wedelolactone, different chromatographic conditions were tried to improve the separation of marker (wedelolactone) from other peaks in suitable time. Wedelolactone was detected and accurately quantified by using RP-HPLC-PDA with methanol: $0.2 \%$ formic acid in water (57:43 $\mathrm{v} / \mathrm{v})$. The retention time $\left(\mathrm{R}_{\mathrm{t}}\right)$ of wedelolactone was found to be $5.9 \mathrm{~min}$ under optimized chromatographic conditions. The specificity of the intended method was established by comparing the retention time and absorption spectra of the target peaks from the analyzed samples with the reference compound. The limit of detection (LOD) and limit of quantification (LOQ) was found to be $0.5 \mu \mathrm{g} / \mathrm{mL}$ and $1 \mu \mathrm{g} / \mathrm{mL}$ for wedelolactone, suggesting the sensitivity of the method. The percent coefficient of variations during the system suitability study was found to be 1.71 and $1.34 \%$ for retention time and response of wedelolactone respectively.

The response for the marker was found to be linear in the range of 5 $100 \mu \mathrm{g} / \mathrm{mL}$ with a coefficient of determination of 0.999 . From the above results, it can be said that wedelolactone showed a broad range of linear detection and was also highly sensitive method. This resulted in a regression equation, $y=31.705 x-32261$ for wedelolactone. Inter and intra-day precision for the quality control samples of wedelolactone was within the acceptance limit of $85-115 \%$. Mean recovery for the quality control samples of markers was found to be within the acceptance limit of $>95 \%$ (98.8\%-wedelolactone). Wedelolactone was found to be stable for $6 \mathrm{~h}$ at ambient temperature and for 15 days below $8^{\circ} \mathrm{C}$. The method was found to be simple, rapid, specific, precise and rugged as the values obtained were within the acceptance limits.

The developed and validated method was applied for the estimation and quantitation of Wedelolactone from methanolic extract of E. alba collected from various geographical regions of India, their different morphological parts and their various herbal and Ayurvedic formulations.

\section{CONCLUSION}

The described HPLC methods represent a linear, accurate, reliable, rapid and effective method for the analysis of wedelolactone from E. alba. This method includes many advantages like high speed detection, excellent peak shapes, less solvent usage, well-defined chemical constituents, detection and quantitation of phytoconstituent (wedelolactone), which is better than fingerprinting. As single marker-based quantitative methods would be complementary approaches for the quality control and stability assessment of herbal preparations, the results of the current study could be used by industries for the characterisation of E. alba and its formulations in order to check their uniformity. The quantitation of phytochemical can help in identifying the best plant part, with reference to the content of phytochemical marker, which in turn will be helpful in commercialization and export of raw materials, herbal extracts and formulations.

These methods can be applied to other plant raw materials containing the same phytochemical markers. This validated method is ready for transfer to any QC laboratory for quality assurance of Ayurvedic formulations. Hence, it can be demonstrated that HPLC is a powerful practical tool for comprehensive quality control of plant raw materials and its formulations. Together these characteristic components can be used as reference markers for identification and authentication of E. alba and as an application of the proposed method it can be used for marker-based standardization of few commercial polyherbal 'Ayurvedic' formulation containing the whole plant of E. alba.

\section{CONFLICT OF INTEREST}

The author declare no conflict of interest.

\section{ACKNOWLEDGEMENT}

The financial assistance from National Medicinal Plants Board, Department of AYUSH, Ministry of Health and Family Welfare, Govt. of India (Project No: Z.18017/187/CSS/R\&D/MH-01/2011-12-NMPB entitled 'Development of HPLC methods for phytochemical markers and their validation as per ICH guidelines with Bioavailability Studies') is highly acknowledged. 


\section{AUTHORS CONTRIBUTION}

DS and GS developed the HPLC analytical method, actively participated in stability studies and validation of the method. All authors read and approved the final manuscript.

\section{REFERENCES}

1. Chopra RN, Chopra IC, Nayar SL. Glossary Of Indian Medicinal Plants. New Delhi: CSIR, 2008. 1956;66-7.

2. Rastogi RP, Mehrotra BN. Compendium Of Indian Medicinal Plants. New Delhi: CSIR, 19912:288.

3. Sharma M, Yusuf M, Hussain S, Hussain A. Phytochemical Constituents And Pharmacological Activities Of Eclipta alba Linn. (Asteraceae): A Review. IRJP. 2012; 3(12):51-53.

4. Chokotia L, Vashistha P, Sironiya R, Matoli H. Pharmacological Activities Of Eclipta alba (L.). Int. J. Res. Dev. Pharm. L. Sci. 2013;2(4):499-502.

5. Sarg TM, Salam NAA, El-Domiaty M, Khafagy SM. The Steroid, Triterpenoid And Flavonoid Constituents Of Eclipta alba (L.) Hassk. (Compositae) Grown In
Egypt. Sci. Pharm. 1981; 49(3):262-4.

6. Saxena AK, Singh B, Anand KK. Hepatoprotective Effects of Eclipta alba On Subcellular Levels In Rats. J Ethnopharmacol. 1993;40(3):155-61.

7. Simonsen HT, Nordskjold JB, Smitt UW, Nyman U, Palpu P, Joshi P, et al. In Vitro Screening Of Indian Medicinal Plants For Antiplasmodial Activity. J Ethnophar macol. 2001;74(2):195-204.

8. Thakur VD, Mengi SA. Neuropharmacological Profile Of Eclipta alba (Linn.) Hassk. J Ethnopharmacol. 2005;102(1):23-31.

9. Handa SS, Sharma A, Chakarborty KK. Natural Products And Plants As Liver Protecting Drugs. Fitoterapia. 1986;57(5):307-51.

10. Murali B, Agrawal A, Anand M.S, Samiulla DS. Estimation Of Wedelolactone And Demethylwedelolactone In Eclipta alba Hassk By Improved Chromatographic Analysis. J. Nat. Remedies. 2002;2(1):99-101.

11. Wagner H, Geyer B, Kiso Y, Hikino H, Rao GS. Coumestans As The Main Active Principles Of The Liver Drugs Eclipta alba And Wedelia calendulacea. Planta Med. 1986;52(5):370-374

12. Kumar S, Dhanani T. Development and validation of a rapid high performance liquid chromatography-photodiode array detection method for estimation of a bioactive compound wedelolactone in extracts of Eclipta alba. Braz. J. Pharm. Sci. 2013:49 (1):57-63.

\section{PICTORIAL ABSTRACT}

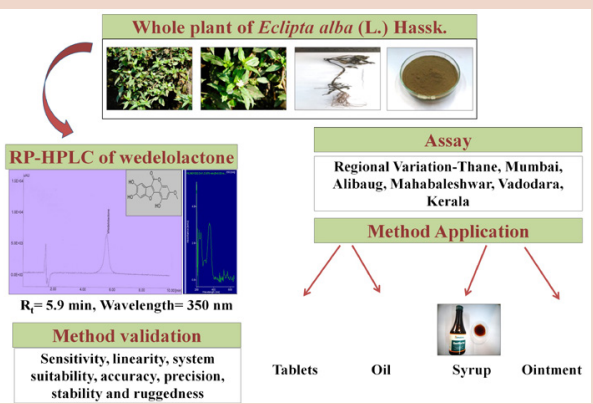

\section{SUMMARY}

- RP-HPLC method was developed for estimation and quantitation of bioactive marker, wedelolactone.

- The developed method was validated as per ICH guidelines in terms of sensitivity, accuracy, system suitability, stability, linearity, ruggedness and precision

- The validated method was applied for the separation and quantitation of wedelolactone from the whole plant of Eclipta alba, its morphological parts, from various regions and from traditional and herbal formulations employing Eclipta alba as one of its ingredients.

- Highest content of wedelolactone was found in the material collected from Alibaug region $(0.0974 \pm 0.0041 \mathrm{mg} / \mathrm{g})$

\section{ABOUT AUTHORS}

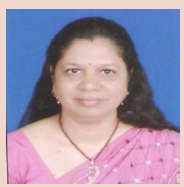

Dr. Sunita Shailajan: Is an Associate Professor in Botany, Incharge of Herbal Research Lab and Scientist Incharge of Animal Testing Centre at Ramnarain Ruia College, University of Mumbai, India. She is recognized research guide in the subject of Botany, Bioanalytical Sciences, Biotechnology and Applied Biology. Her specialization is in the area of standardization of herbal raw materials and ASU formulations, instrumentation (HPTLC and HPLC), bioanalytical method validation, pharmacokinetic studies. She has more than 90 scientific publications and made presentations in various National and International conferences in India and overseas. She has been working as Principal Investigator for various Government funded projects. 